\title{
Proton Conductivity via Trapped Water in Phosphonate-based Metal-Organic Frameworks Synthesized in Aqueous Media
}

Unjila Afrin, ${ }^{1}$ Mohammad Rasel Mian, ${ }^{1}$ Ken-ichi Otake, ${ }^{3}$ Riki Drout, ${ }^{1}$ Louis R. Redfern, ${ }^{1}$ Satoshi Horike, ${ }^{3}$ Timur Islamoglu, ${ }^{1}$ Omar K. Farha $* 1,2$

${ }^{1}$ Department of Chemistry and International Institute of Nanotechnology, ${ }^{2}$ Department of Chemical \& Biological Engineering, Northwestern University, 2145 Sheridan Road, Evanston, IL 60208, United States.

${ }^{3}$ Institute for Integrated Cell-Material Sciences (iCeMS), Kyoto University Institute for Advanced Study (KUIAS), Kyoto University, Yoshida Ushinomiya-cho, Sakyo-ku, Kyoto 606-8501, Japan

\section{Table of Contents}

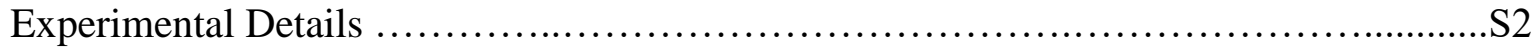

Crystal data and structure refinement for Mg-NU-225 and Fe-NU-225 ...............S3

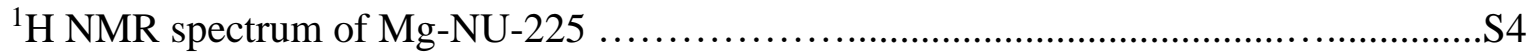

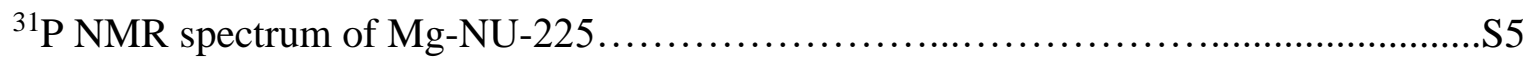

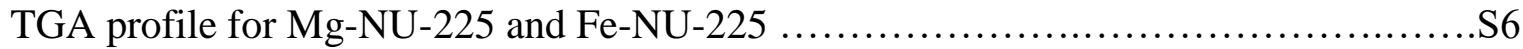

DRIFTS for Glyphosate, Mg-NU-225 and Fe-NU-225 ..........................S7

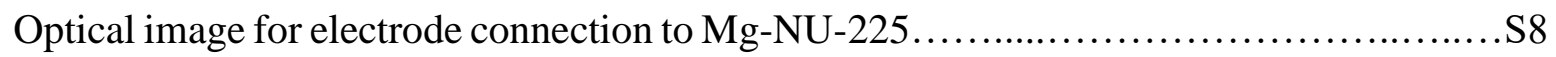

Humidity dependence proton conductivity of Mg-NU-225 along the plane.............S9

Humidity dependence proton conductivity of Fe-NU-225 along the plane...............S9

The Arrhenius plot of the proton conductivities perpendicular to plane.................S10

PXRD patterns of Mg-NU-225 at different RH............................

References..................................................................... 


\section{$\underline{\text { Experiment Details }}$}

\section{Characterization and Instrumentation}

X-ray diffraction analysis: PXRD data were collected at the IMSERC X-ray Facility at Northwestern University on a STOE-STADI-MP powder diffractometer equipped with an asymmetric curved Germanium monochromator $\left(\mathrm{CuK}_{\alpha 1}\right.$ radiation, $\left.\lambda=1.54056 \AA\right)$ and one dimensional silicon strip detector (MYTHEN2 1K from DECTRIS). The line focused Cu Xray tube was operated at $40 \mathrm{kV}$ and $40 \mathrm{~mA}$. Powder was packed in a $3 \mathrm{~mm}$ metallic mask and sandwiched between two layers of polyimide tape. Intensity data from 2 to 30 degrees $2 \theta$ were collected over a period of 10-20 min. The instrument was calibrated against a NIST Silicon standard (640d) prior the measurement.

X-ray photoelectron spectroscopy (XPS): XPS measurements were carried out at the KECK-II/NUANCE facility at Northwestern University on a Thermo Scientific ESCALAB $250 \mathrm{Xi}(\mathrm{Al} \mathrm{K} \alpha$ radiation, $h v=1486.6 \mathrm{eV}$ ) equipped with an electron flood gun. All spectra from XPS were analyzed as reference to the $\mathrm{C} 1 \mathrm{~s}$ peak $(284.8 \mathrm{eV})$ by using Thermo Scientific Advantage Data System software.

\section{Diffuse Reflectance Infrared Fourier Transform Spectroscopy (DRIFTS): DRIFTS} spectra were recorded on a Nicolet 6700 FTIR spectrometer equipped with an MCT detector. The detector was cooled with liquid $\mathrm{N}_{2}$, and the spectra were collected under an $\mathrm{Ar}$ atmosphere. $\mathrm{KBr}$ was utilized as a background spectrum.

Single-Crystal X-ray Structural Determination: The data for Mg-NU-225 and FeNU-225 were collected at $100 \mathrm{~K}$ on a 'Bruker APEX-II CCD' diffractometer with a MoK $\alpha(\lambda$ $=0.71073 \AA$ ) microfocus X-ray source. The single crystal was mounted on MicroMesh (MiTeGen) with paratone oil. The structures were determined by intrinsic phasing (SHELXT 2018/2) S1 $^{\mathrm{S}}$ and refined by full-matrix least-squares refinement (SHELXL-2018/3) ${ }^{\mathrm{S} 2}$ using the Olex $2^{\mathrm{S} 3}$ software packages. The refinement results are summarized in Table S1. Crystallographic data for these structures in CIF format have been deposited in the Cambridge Crystallographic Data Centre (CCDC) under deposition numbers CCDC2040932 and 2040933 for Mg-NU-225 and Fe-NU-225, respectively. The data can be 
obtained free of charge via www.ccdc.cam.ac.uk/data_request/cif (or from the Cambridge Crystallographic Data Centre, 12 Union Road, Cambridge CB2 1EZ, U.K.).

${ }^{31} \mathbf{P}$ NMR spectroscopy: NMR spectra for hydrolysis profiles of simulant were collected on the Bruker Advance III $600 \mathrm{MHz}$ system with three RF channels at IMSERC (Integrated Molecular Structure Education and Research Center) of Northwestern University.

Table S1 Crystal data and structure refinement for Mg-NU-225 and Fe-NU-225.

\begin{tabular}{|c|c|c|}
\hline & Mg-NU-225 & Fe-NU-225 \\
\hline Empirical formula & $\mathrm{C}_{6} \mathrm{H}_{14} \mathrm{MgN}_{2} \mathrm{O}_{10} \mathrm{P}_{2}$ & $\mathrm{C}_{6} \mathrm{H}_{14} \mathrm{FeN}_{2} \mathrm{O}_{10} \mathrm{P}_{2}$ \\
\hline Formula weight & 360.44 & 391.98 \\
\hline Temperature/K & 100.15 & 100.15 \\
\hline Crystal system & monoclinic & monoclinic \\
\hline Space group & $\mathrm{C} 2 / \mathrm{c}$ & $\mathrm{C} 2 / \mathrm{c}$ \\
\hline $\mathrm{a} / \AA ̊$ & $7.2864(4)$ & $7.2947(4)$ \\
\hline $\mathrm{b} / \AA$ & $16.6379(9)$ & $16.6338(10)$ \\
\hline $\mathrm{c} / \AA ̊$ & $9.9732(5)$ & $10.0033(6)$ \\
\hline$\alpha^{\circ}$ & 90 & 90 \\
\hline$\beta /{ }^{\circ}-\beta$ & $92.893(3)$ & $93.610(2)$ \\
\hline$\gamma /{ }^{\circ}$ & 90 & 90 \\
\hline Volume $/ \AA^{3}$ & $1207.51(11)$ & $1211.38(12)$ \\
\hline $\mathrm{Z}$ & 4 & 4 \\
\hline$\rho_{\text {calc }} / \mathrm{cm}^{3}$ & 1.983 & 2.149 \\
\hline$\mu / \mathrm{mm}^{-1}$ & 0.473 & 1.569 \\
\hline $\mathrm{F}(000)$ & 744.0 & 800.0 \\
\hline Crystal size $/ \mathrm{mm}^{3}$ & $0.4 \times 0.2 \times 0.1$ & $0.3 \times 0.15 \times 0.15$ \\
\hline Radiation & $\operatorname{MoK} \alpha(\lambda=0.71073)$ & $\operatorname{MoK} \alpha(\lambda=0.71073)$ \\
\hline $2 \Theta$ range for data collection $/{ }^{\circ}$ & 4.896 to 59.01 & 6.376 to 88.64 \\
\hline Index ranges & $\begin{array}{l}-8 \leq \mathrm{h} \leq 10,-22 \leq \mathrm{k} \leq 22,-13 \\
\leq 1 \leq 13\end{array}$ & $\begin{array}{l}-14 \leq \mathrm{h} \leq 13,-22 \leq \mathrm{k} \leq 32,-19 \\
\leq 1 \leq 11\end{array}$ \\
\hline Reflections collected & 6094 & 9916 \\
\hline Independent reflections & $\begin{array}{l}1666\left[R_{\text {int }}=0.0335, R_{\text {sigma }}=\right. \\
0.0297]\end{array}$ & $\begin{array}{l}4637\left[R_{\text {int }}=0.0311, R_{\text {sigma }}=\right. \\
0.0445]\end{array}$ \\
\hline Data/restraints/parameters & $1666 / 0 / 103$ & $4637 / 0 / 103$ \\
\hline Goodness-of-fit on $\mathrm{F}^{2}$ & 1.119 & 1.076 \\
\hline
\end{tabular}


Final $R$ indexes $[\mathrm{I}>=2 \sigma(\mathrm{I})] \quad \mathrm{R}_{1}=0.0298, \mathrm{wR}_{2}=0.0786 \quad \mathrm{R}_{1}=0.0365, \mathrm{wR}_{2}=0.0932$

Final $\mathrm{R}$ indexes [all data] $\quad \mathrm{R}_{1}=0.0345, \mathrm{wR}_{2}=0.0813 \quad \mathrm{R}_{1}=0.0429, \mathrm{wR}_{2}=0.0963$

Largest diff. peak/hole / e $\AA^{-3} \quad 0.53 /-0.47 \quad 1.93 /-1.56$

\section{${ }^{1} \mathrm{H}$ NMR spectra of Mg-NU-225}

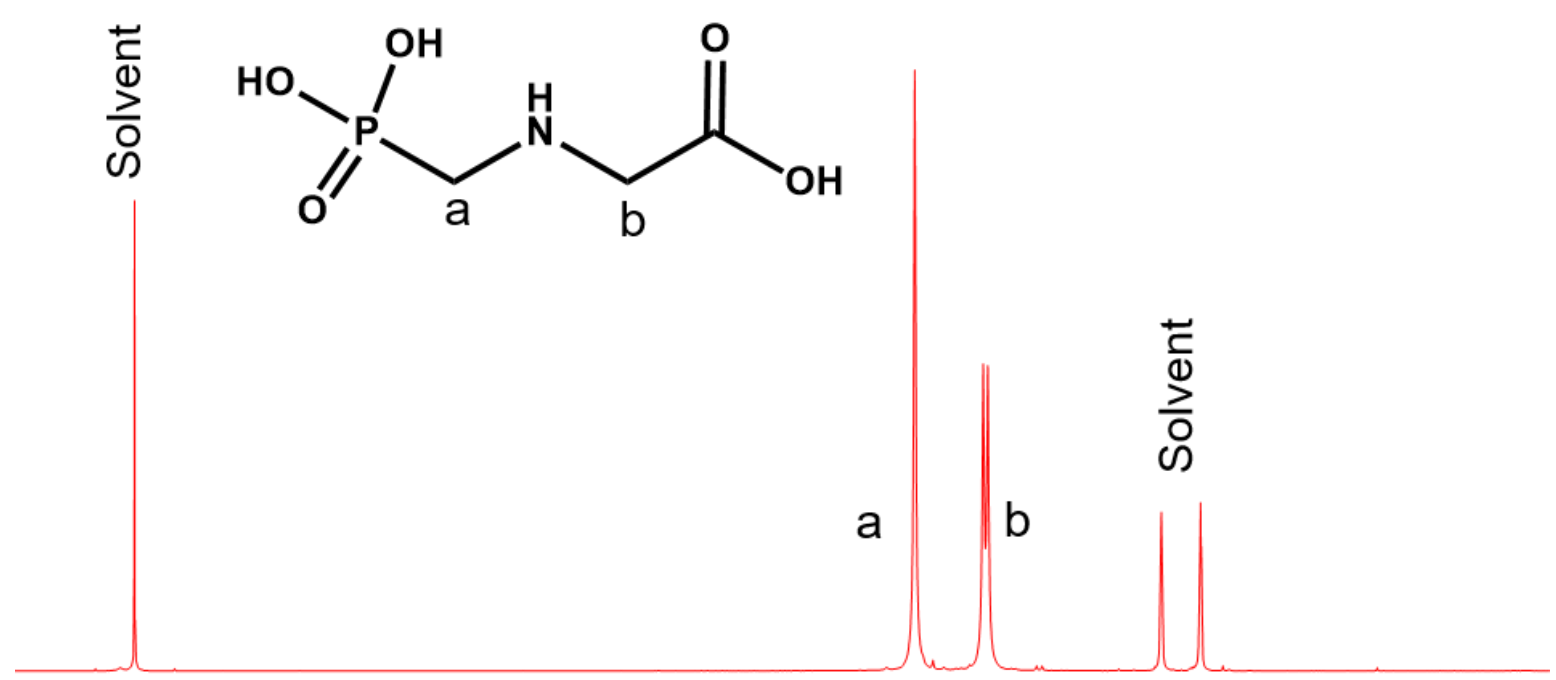

$\begin{array}{llllllllllllllll}8.5 & 8.0 & 7.5 & 7.0 & 6.5 & 6.0 & 5.5 & 5.0 & 4.5 & 4.0 & 3.5 & 3.0 & 2.5 & 2.0 & 1.5 & 1\end{array}$ f1 (ppm)

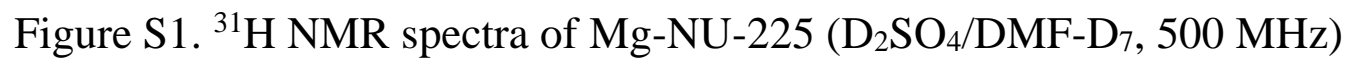


${ }^{31} \mathrm{P}$ NMR spectra of Mg-NU-225

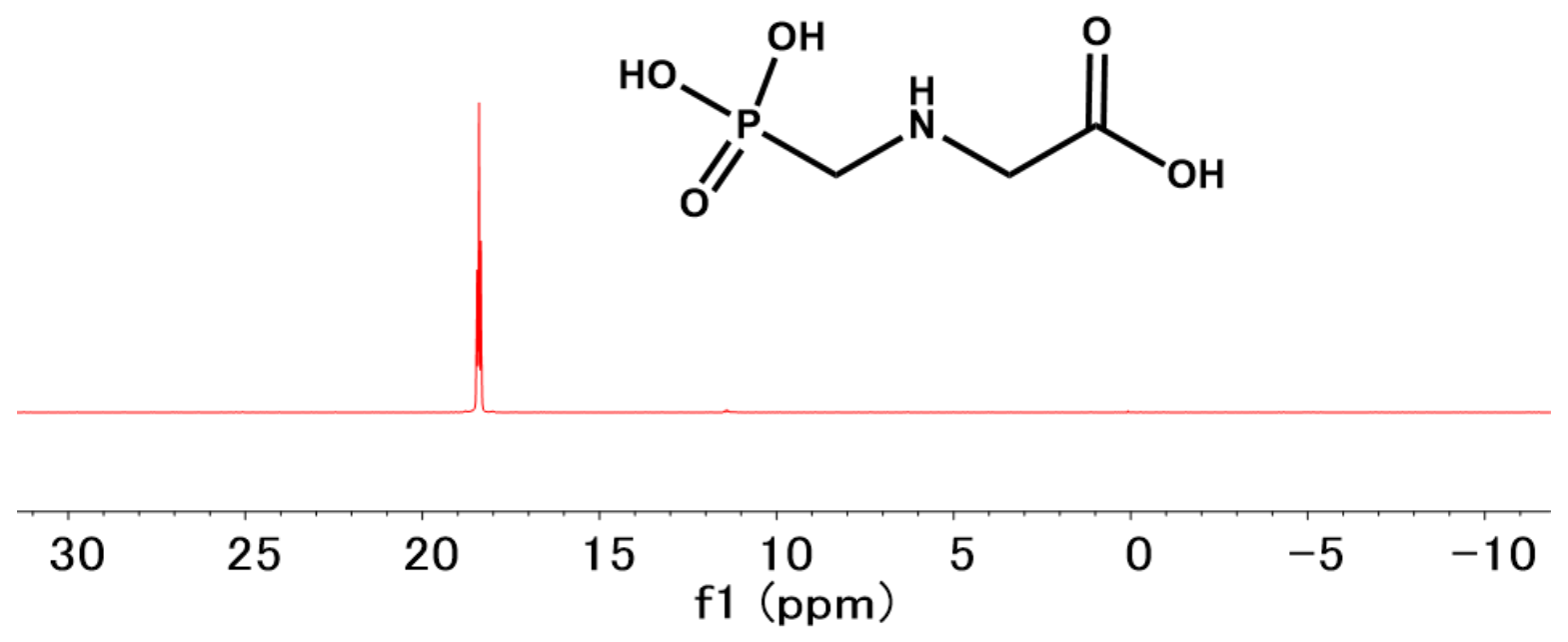

Figure S2. ${ }^{31} \mathrm{P}$ NMR spectra of Mg-NU-225 (D $\left.\mathrm{SO}_{4} / \mathrm{DMF}^{-\mathrm{D}_{7},} 500 \mathrm{MHz}\right)$ 
Thermogravimetric analysis of Mg-NU-225 and Fe-NU-225

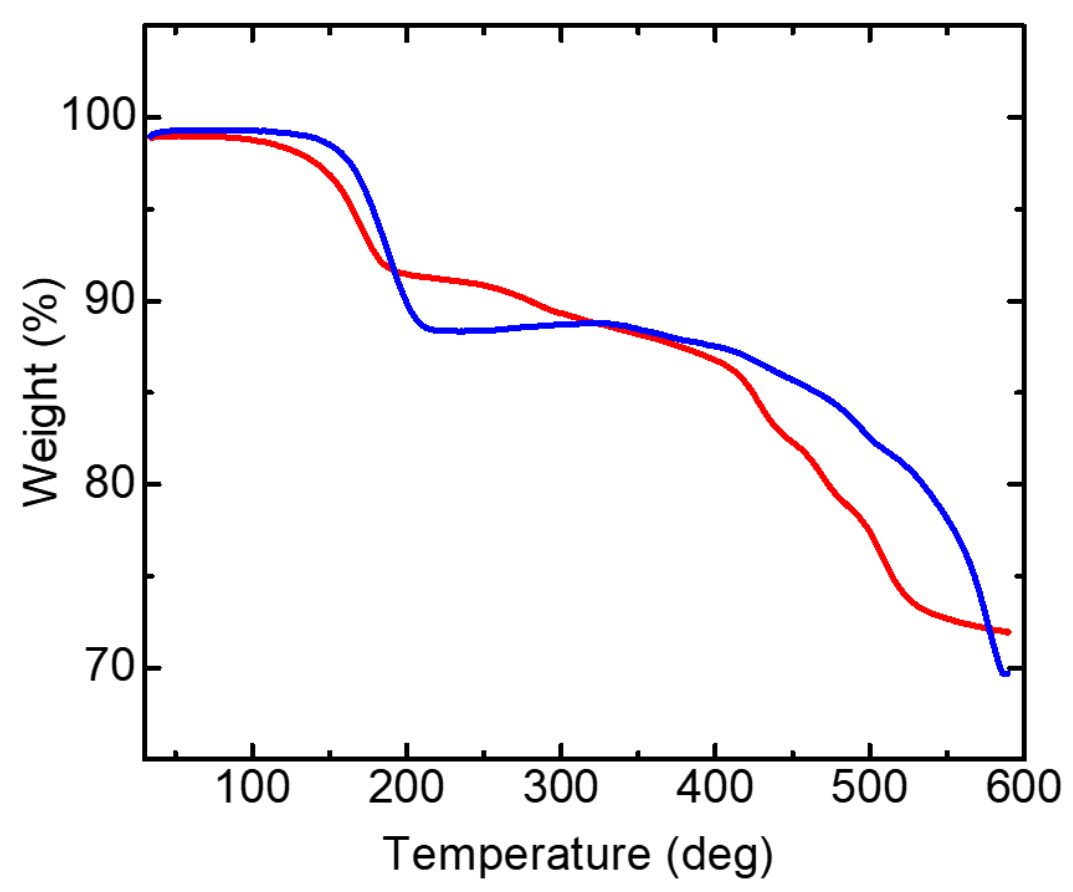

Figure S3 TGA profile of Mg-NU-225 (blue) and Fe-NU-225 (red) under N2 condition with a 10 degree/min rate. 
IR spectra for Mg-NU-225, Fe-NU-225 and glyphosate

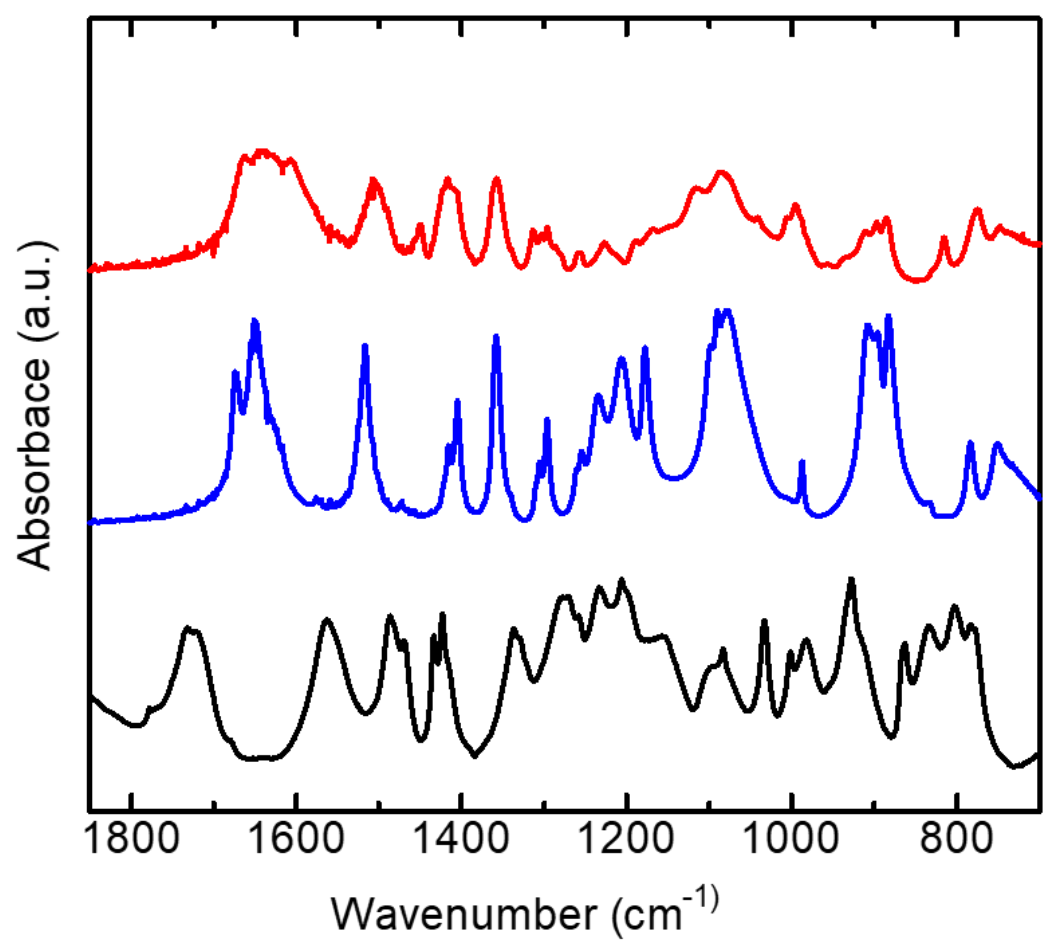

Figure S4 DRIFTS spectra of Fe-NU-225 (top), Mg-NU-225 (middle) and glyphosate (bottom) 


\section{Optical image for electrode connection to Mg-NU-225}

a)

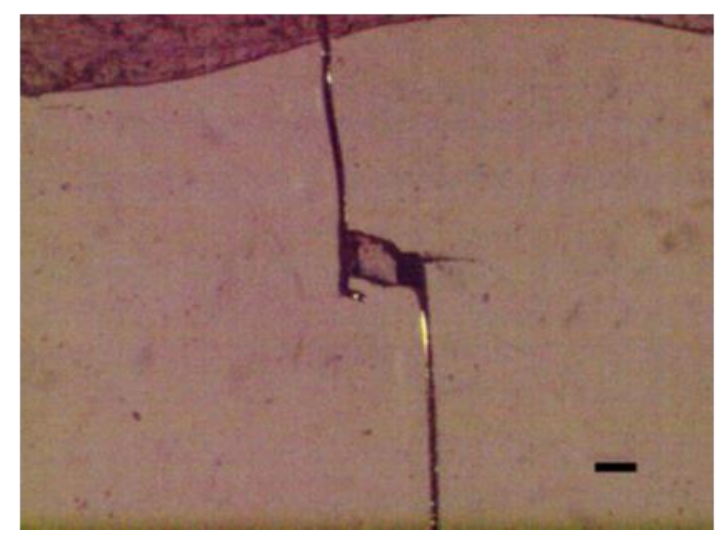

b)

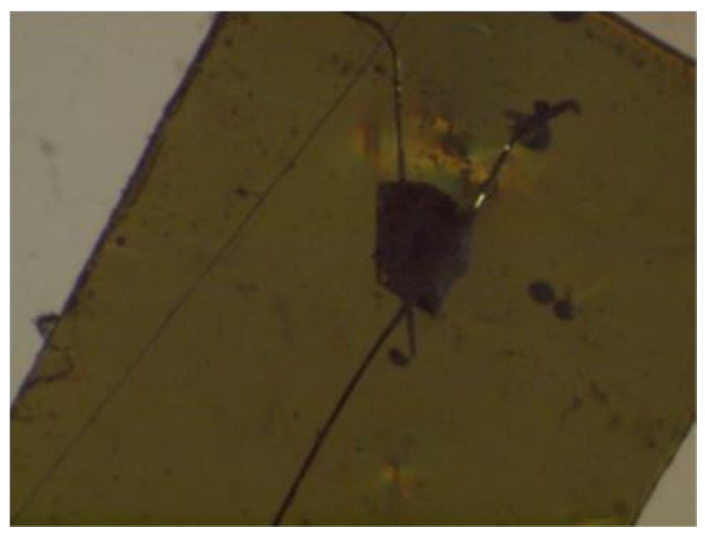

Figure S5 Representative set up using two-contact probe method on a single crystal with two gold wires connected to the edges of the crystal by silver paste. Gold electrode attached to Mg-NU-225 along the plane direction with a crystal dimension $0.920 \times 0.46 \times 0.30 \mathrm{~mm}^{3}$ (a) and perpendicular to the plane with a dimension $0.920 \times 0.31 \times 0.10 \mathrm{~mm}^{3}$ (b). 


\section{Humidity dependence proton conductivity of Mg-NU-225 along the plane}

a)

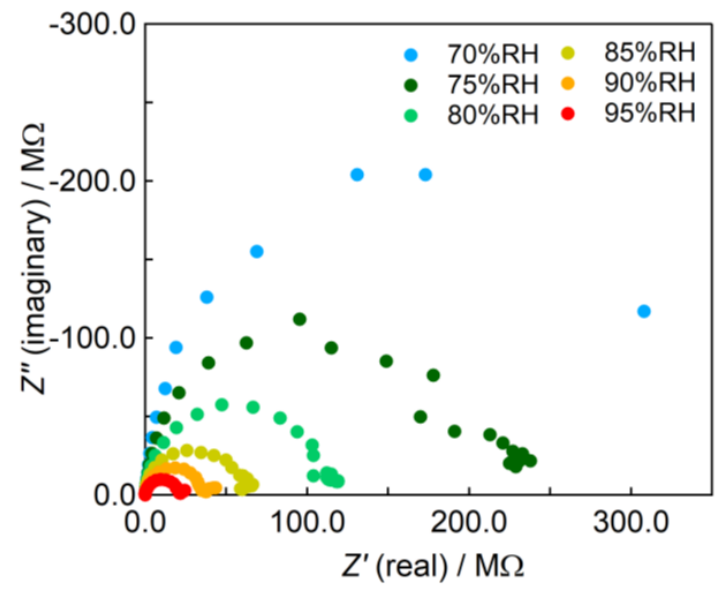

b)

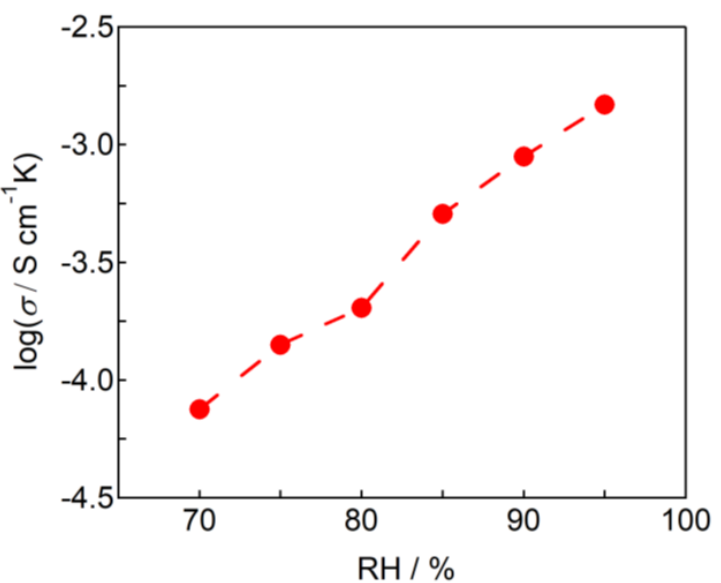

Figure S6 (a) Nyquist plot for Mg-NU-225 at different relative humidity (RH) levels. (b) Conductivity of Mg-NU-225 plotted versus relative humidity.

\section{Humidity dependence proton conductivity of Fe-NU-225 along the plane}

a)

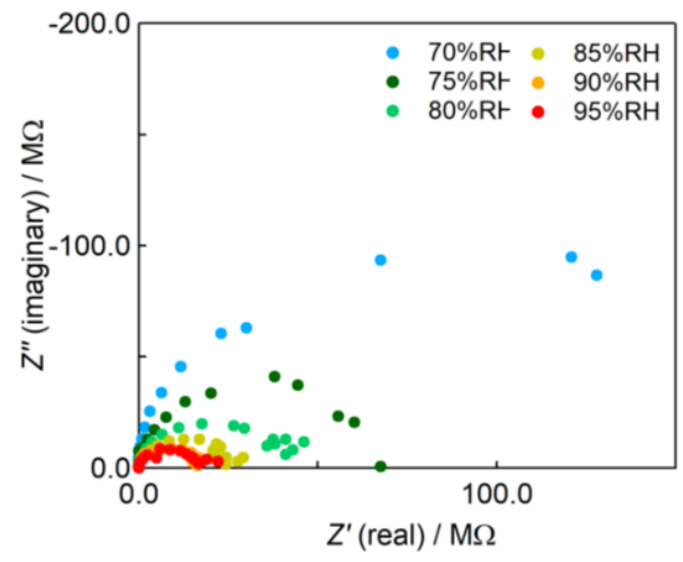

b)

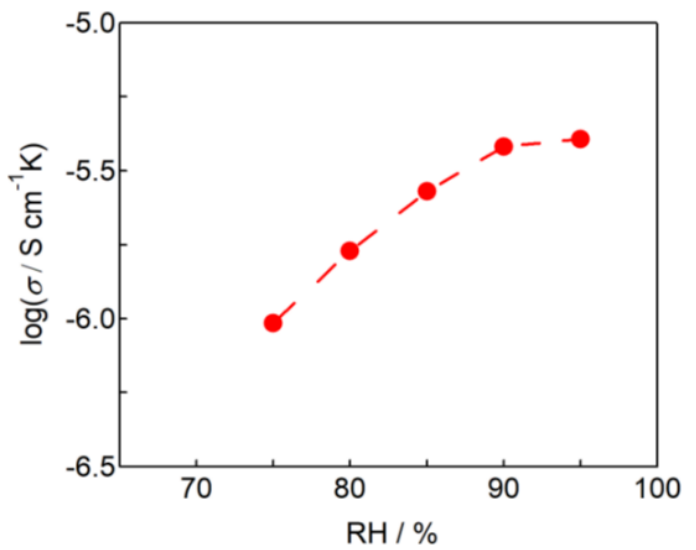

Figure S7 (a) Nyquist plot for Fe-NU-225 at different relative humidity (RH) levels. (b) Conductivity of Fe-NU-225 plotted versus relative humidity. 
The Arrhenius plot of the proton conductivities perpendicular to plane

a)

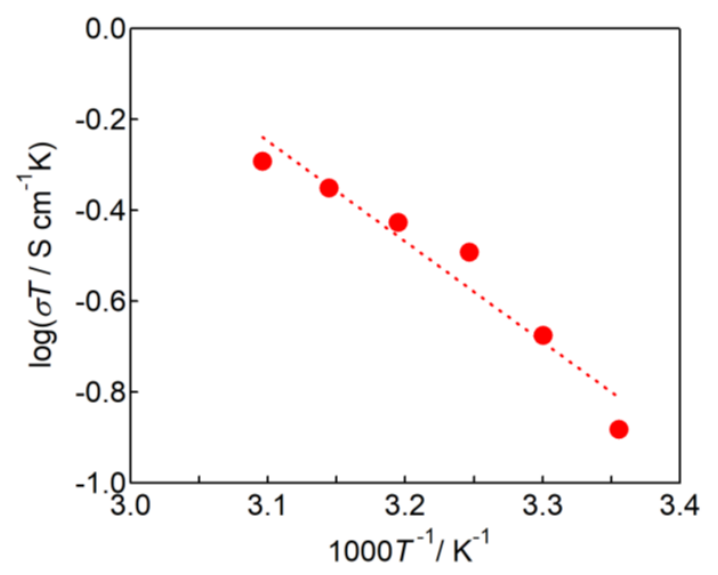

b)

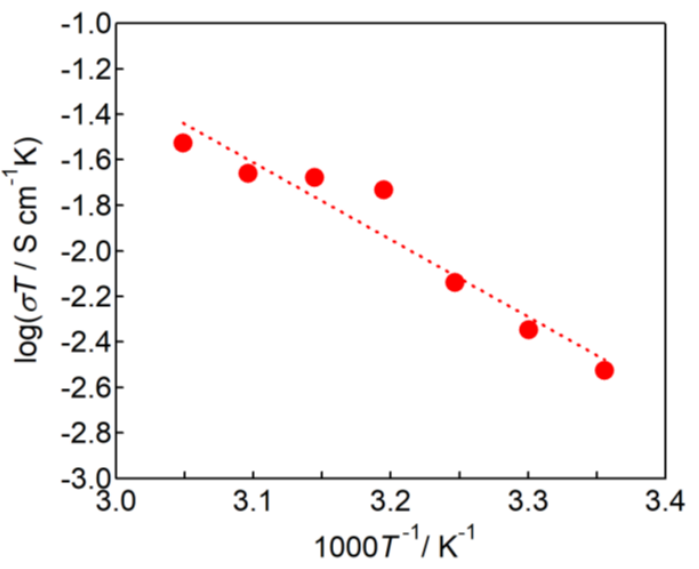

Figure S8 Arrhenius plot for proton conductivity of Mg-NU-225 (a) and Fe-NU-225 (b)

\section{PXRD patterns of Mg-NU-225 at different RH}

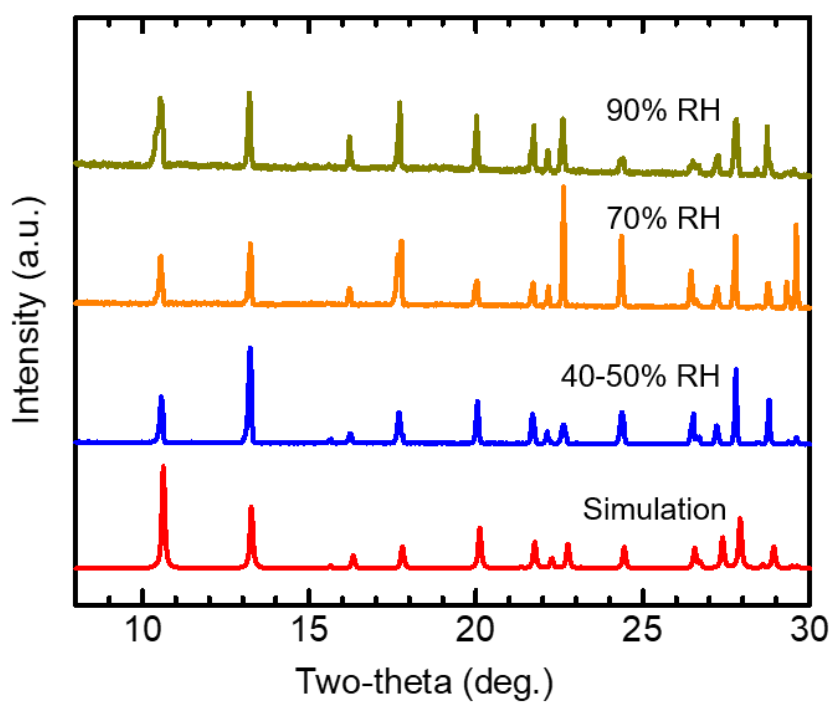

Figure S9 PXRD patterns of Mg-NU-225 at different relative humidity $(\mathrm{RH})$ levels. 


\section{Reference.}

S1) Sheldrick, G. SHELXT -Integrated space-group and crystal-structure determination. Acta Crystallogr A 2015, 71, 3-8.

S2) Sheldrick, G.Crystal structure refinement with SHELXL. Acta Crystallographica Section C 2015, 71, 3-8.

S3) Dolomanov, O. V.; Bourhis, L. J.; Gildea, R. J.; Howard, J. A. K.; Puschmann, H. OLEX2: a complete structure solution, refinement and analysis program. J. Appl.

Crystallogr. 2009, 42, 339-341. 